\title{
Linee Guida Operative per la diagnosi delle Parassitosi Intestinali
}

\author{
Francesco Bernieri, Pietro Casella, Daniele Crotti, Vincenzo Cutrupi, Daniela Galli, \\ Luigi Di Matteo, Annibale Raglio
}

Comitato di Studio per la Parassitologia dell'Associazione Microbiologi Clinici Italiani (AMCLI-CoSP)

\section{INDICE}

PRESENTAZIONE

INTRODUZIONE

1. L'ESAME COPROPARASSITOLOGICO

2. PROCEDURE DIAGNOSTICHE \& LINEE GUIDA OPERATIVE

3. TEMPI ANALITICI \& REFERTAZIONE APPENDICE

BIBLIOGRAFIA

ALLEGATI

LEGENDA: Le raccomandazioni sono riportate all'interno di un rettangolo, seguite da una lettera maiuscola per esprimere la Forza della Raccomandazione e da un numero romano che corrisponde al Livello delle Prove, entrambi posti tra parentesi e evidenziati su sfondo grigio.

\section{PRESENTAZIONE}

Il Comitato di Studio per la Parassitologia dell'AMCLI si occupa da oltre 10 anni di aggiornamento e diffusione della parassitologia di interesse medico all'interno dell'Associazione, attraverso pubblicazioni e corsi.

La lunga esperienza di corsi di parassitologia sia "di base" che "avanzati" ha permesso al Comitato di entrare in contatto con laboratoristi di tutta Italia e con le loro richieste, prima tra tutte quella di fornire delle indicazioni pratiche per il laboratorista che inizia ad occuparsi di parassitologia e deve scegliere le tecniche diagnostiche da utilizzare.

Da queste numerosissime e pressanti richieste è nata la decisione di pubblicare delle Linee Guida Operative (LGO) per la ricerca dei parassiti intestinali, indirizzate al laboratorista, medico, biologo o tecnico di laboratorio, che si occupa di parassitologia.

Tale lavoro è stato anche motivato dalla mancanza di Linee Guida italiane ufficiali per lo specifico argomento.

Obiettivi

- Aumentare l'efficacia dell'esame coproparassitologico finalizzato alla ricerca dei parassiti intestinali.

- Diffondere l'uso di tecniche idonee ad ottenere i risultati analitici migliori. $\underline{\text { Raccomandazioni }}$

- Quali tecniche utilizzare per effettuare un Esame Coproparassitologico Standard.

- Effettuare sempre la Concentrazione Formolo Etere - Etil Acetato su ogni campione fecale da sottoporre a ricerca dei parassiti nelle feci.

- Effettuare sempre una colorazione permanente quale necessario completamento della ricerca dei parassiti nelle feci.

- Conoscere i limiti della Concentrazione e le indicazioni per ricerche mirate di alcuni parassiti (Strongyloides stercoralis, Enterobius vermicularis, Cryptosporidium parvum, Dientamoeba fragilis).

- Cosa riportare su un referto coproparassitologico.

\section{Metodologia}

In Appendice si riportano gli schemi classificativi per la Forza della raccomandazione ed il Livello di prova nonché la metodologia seguita nel lavoro.

\section{INTRODUZIONE}

Le parassitosi intestinali sono causate da protozoi (microrganismi unicellulari eucarioti appartenenti al Regno Protoctista, Sottoregno Protozoa) e da elminti o vermi (organismi pluricellulari appartenenti al Regno Animalia) (1, pag. 99, pag. 181).

La diagnostica parassitologica si basa soprattutto sulla microscopia (1, pag. 349; 2, pag. 2-1, 2-47; 3, Introduction; 4, pag. 1).

L'identificazione dei parassiti si effettua utilizzando principalmente criteri di tipo morfologico (5, pag. 3-7).

Le forme diagnostiche sono rappresentate da trofozoiti, cisti, oocisti e spore di Protozoi e da uova, larve, proglottidi e adulti di Elminti; è quindi di grande importanza la corretta conservazione di tali forme (1, pag. 349; 3, Introduction; 4, pag. 3-7; 6, pag. 9 e 32; 7, pag. 173 e 189). Di conseguenza, rivestono un ruolo chiave la raccolta dei campioni ed il loro trattamento con fissativi quali Formalina al 10\%, SAF, PVA, MIF o altri.

Per ottenere campioni idonei da sottoporre ad esame è necessario fornire dettagliate istruzioni scritte ai pazienti (1, pag. 349; 6, pag. 9).

In Allegato 1 , che non è parte integrante di queste LGO, si riportano esempi di istruzioni per la raccolta delle feci per l'esame coproparassitologico, per l'esecuzione dello Scotch Test e per la ricerca 
di Strongyloides stercoralis (tabelle 1, 2 e 3).

Ogni laboratorio deve decidere quali esami effettuare ed in base a ciò preparare le istruzioni da fornire ai pazienti. Infatti, per l'esame coproparassitologico è possibile accettare feci "fresche" oppure feci già fissate, in relazione alle tecniche che si intendono utilizzare (2, pag. 2-3, 2-4, 2-5).

Ai fini di una corretta diagnosi, può essere necessario analizzare più campioni fecali ( $\mathrm{da} 2$ a 6, mediamente 3), possibilmente raccolti a giorni alterni (1, pag. 349; 6, pag. 9; 8, pag. $491 ; 9 ; 10 ; 11 ; 13 ; 14 ; 15 ; 16 ; 17 ; 18$, pag. 25$)$ $\rightarrow(\mathbf{A}, \mathbf{V I})$.

\section{L'ESAME COPROPARASSITOLOGICO}

In presenza di richiesta generica di "ricerca parassiti nelle feci", occorre effettuare un esame coproparassitologico per ricercare sia trofozoiti, cisti ed oocisti di protozoi sia uova e larve di elminti (1, pag. 348; 3, Introduction; 6 , pag. 9; 18, pag. 6; 19, pag. 741-785) $\rightarrow(\mathrm{A}, \mathrm{VI})$.

Si intende per "esame coproparassitologico" l'insieme delle tecniche che si utilizzano per ricercare i parassiti intestinali in campioni di feci. Alcune tecniche sono in grado di evidenziare sia protozoi che elminti, altre tecniche sono adatte alla ricerca di un solo gruppo di parassiti o di una singola specie.

Gli esami riportati di seguito rappresentano le principali tecniche per la ricerca dei parassiti intestinali con brevi indicazioni sul loro utilizzo. Per una più ampia trattazione e per le specifiche metodiche si rimanda a specifici testi $(\mathbf{1} ; \mathbf{2} ; \mathbf{3} ; \mathbf{4}$; $5 ; 6 ; 7 ; 8 ; 18 ; 19 ; 36$ )

- Esame macroscopico: utile per evidenziare elminti e/o parti di essi; permette di segnalare i campioni diarroici.

- Esame microscopico diretto: utile per evidenziare sia gli elminti che i protozoi; evidenzia soprattutto i trofozoiti dei protozoi.

- Esame dopo concentrazione: utile per evidenziare sia gli elminti che i protozoi.

- Scotch Test: utile per evidenziare E. vermicularis (talora anche Taenia spp).

- Colorazione Tricromica: utile per evidenziare tutti i protozoi (coccidi esclusi).

- Colorazione Ematossilina ferrica: utile per evidenziare tutti i protozoi (coccidi esclusi).

- Colorazione di Giemsa: utile per evidenziare G. intestinalis e D. fragilis.

- Metodo di Baermann: utile per evidenziare le larve di S. stercoralis.
- Coltura su agar delle larve: utile per evidenziare le larve di S. stercoralis, $N$. americanus, A. duodenale, Trichostrongylus spp.

- Colorazioni all'Alcool Acido resistenza: utili per evidenziare le oocisti di C. parvum e degli altri coccidi.

- Colorazione Tricromica modificata: utile per evidenziare le spore dei microsporidi.

- Ricerca di coproantigeni (EIA o Immunofluorescenza): utili per evidenziare la presenza di C. parvum e G. intestinalis

Un Esame coproparassitologico standard deve consistere almeno di un esame microscopico diretto, un esame microscopico dopo concentrazione ed una colorazione permanente, insieme che corrisponde all'anglosassone "Ova \& Parasites" (1, pag. 348 - 351; 3, Introduction; 4, pag. 8 - 27, 82; 6, pag. 14 17 ; 7 pag. 178 e $348 ; 10 ; 11 ; 13 ; 18$, pag. 42 $43 ; 20 ; 21) \rightarrow(A, V I)$.

Il laboratorio che vuole cimentarsi nella diagnostica delle parassitosi intestinali deve essere almeno in grado di eseguire 1' Esame Coproparassitologico Standard e lo Scotch Test (1, pag. 348 - 351, 357; 3, Introduction; 4, pag. 38; 6, pag. $14-17$ e 24; 7, pag. 178; 10; $11 ; 12 ; 20 ; 21 ; 22 ; 23) \rightarrow(A, V)$.

Tali raccomandazioni si basano sulle seguenti osservazioni:

1. la possibilità dell'esame diretto "a fresco", effettuato sulle feci diarroiche, di evidenziare i trofozoiti dei protozoi ed il loro eventuale movimento (1, pag. 350; 6, pag. 14 - 15; 20; 21)

2. la capacità delle tecniche di concentrazione di evidenziare sia protozoi che elminti con una sensibilità molto più elevata di quella dell'esame diretto (3, Introduction; 4, pag. 11; 6, pag. $16 ; 7$. pag. $184 ; 10 ; 12 ; 20 ; 21$ )

3. la capacità delle colorazioni permanenti (Tricromica, Ematossilina ferrica, Giemsa) di evidenziare Dientamoeba fragilis (non evidenziabile con le 2 tecniche precedenti) e di permettere (Tricromica, Ematossilina ferrica) l'identificazione dei trofozoiti dei protozoi a livello di specie (3, Introduction; 4, pag. 17$25 ; 8$, pag. $160 ; 10 ; 12 ; 24 ; 25 ; 26 ; 27 ; 28$, pag. 42).

4. l'aggiunta dello Scotch Test è motivata dal fatto che gli ossiuri, evidenziabili solo occasionalmente attraverso l'esame coproparassitologico standard, rappresentano l'elmintiasi più diffusa in Italia $(\mathbf{2 2} ; \mathbf{2 3})$. 
Esistono numerose tecniche per effettuare la concentrazione. Il Comitato di Studio per la Parassitologia consiglia l'uso di una tecnica che concentri per sedimentazione (centrifugazione) ed in particolare della Concentrazione Formolo Etere o Etil Acetato (FEA) (1, pag. 350; 3, Plate 2; 4, pag. 11; 6, pag. 16 - 17; 7, pag. 185; 8, pag. 495; 18, pag. 27-28; 20$) \rightarrow(A, V I)$.

\section{PROCEDURE DIAGNOSTICHE \& LINEE-GUIDA OPERATIVE}

\section{ESAME COPROPARASSITOLOGICO STANDARD: \\ Flow chart 1}

\section{Il laboratorio accetta campioni fecali "freschi", non fissati}

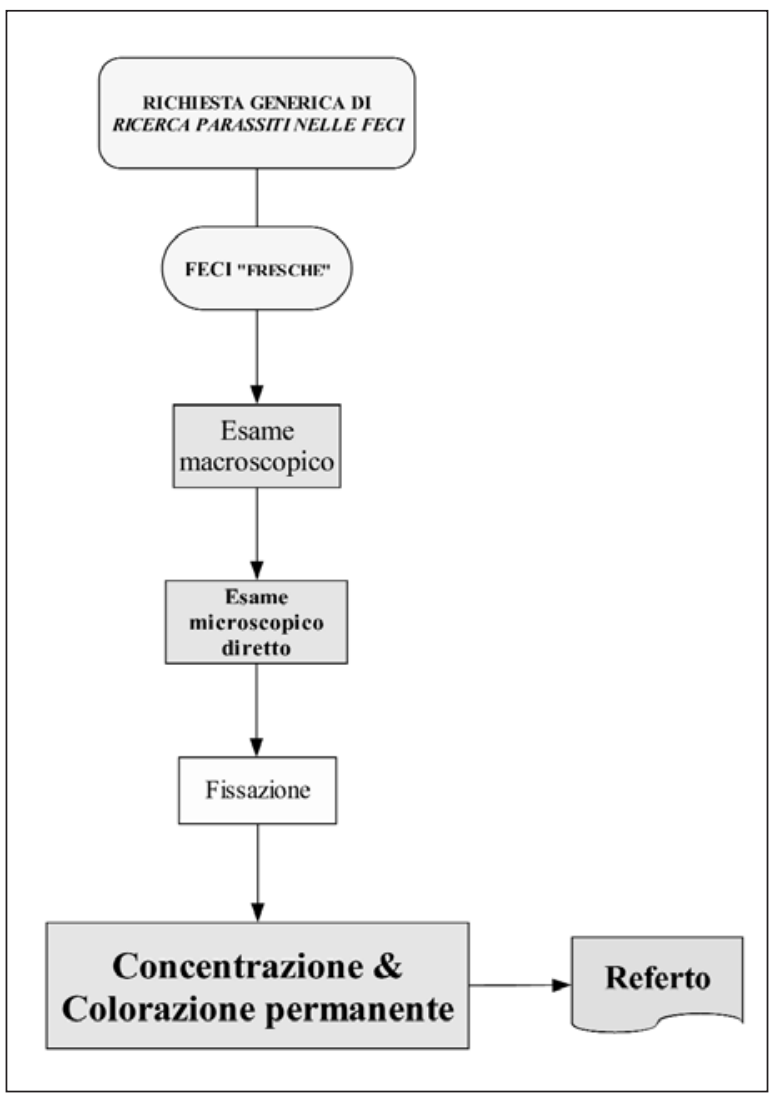

L'insieme di Esame Coproparassitologico Standard e Scotch Test permette di effettuare una buona diagnostica di base ma non è sufficiente ad evidenziare alcuni parassiti intestinali per i quali occorrono tecniche specifiche (1, pag. 345-359; 2, pag. 2-49 - 2-52; 4, pag. 25-34; 7, pag. 178). E' questo il caso della ricerca di Strongyloides stercoralis e di Cryptosporidium parvum, per $\mathrm{i}$ quali sono necessarie indagini mirate, quali:

- Metodo di Baermann e/o coltura su agar per la
ESAME COPROPARASSITOLOGICO STANDARD:

Flow chart 2

Il laboratorio accetta campioni fecali già opportunamente fissati

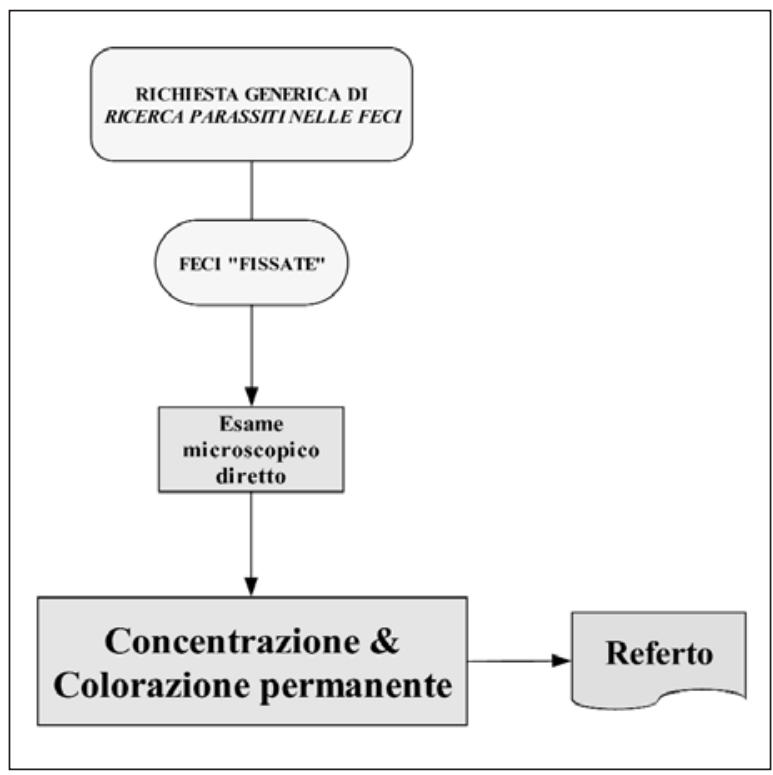

ricerca di Strongyloides stercoralis (1, pag. 354; 2, pag. 2-52 - 2-56; 4 pag. 32; 7, pag. 191; $29 ; 30 ; 31 ; 32 ; 33 ; 34)$.

- Colorazioni all'alcool acido resistenza per la ricerca delle oocisti di Cryptosporidium parvum (1, pag. 352; 2, pag. 2-37, 2-38; 3, Introduction; 4, pag. 25 - 30; 6, pag. 17-18; 8, pag. $385 ; 17 ; 24 ; 35$ ).

L'esame coproparassitologico standard è da considerarsi il minimo che deve essere garantito per una corretta diagnostica parassitologica di base: se un laboratorio non è in grado di fornire tale garanzia non dovrebbe eseguire esami coproparassitologici (1, pag. 348-351; 3, Introduction; 6, pag. 14$17 ; 7$, pag. 178; 10; 11; 20; $21 ; 22 ; 23) \rightarrow(A, V)$.

\section{TEMPI ANALITICI \& REFERTAZIONE}

Tempi analitici

Tempi di fissazione dei campioni biologici: seguire le istruzioni riportate dal produttore o da manuali di riferimento. Una corretta fissazione è importante per la conservazione delle forme diagnostiche e per la sicurezza dell'operatore.

Tempi di esecuzione delle metodiche: seguire le istruzioni dei kit commerciali o di manuali di riferimento.

Lettura dei sedimenti ottenuti da concentra- 
zione: esame microscopico dell'intero preparato; almeno 9 minuti di lettura per vetrino a $10 \mathrm{X}$ ed a 40X (3, Plate 1; 12).

Lettura delle colorazioni permanenti: esame microscopico dell'intero preparato; lettura di almeno 300 campi a 100X; almeno 12 minuti di lettura per vetrino $(6$, pag. $23-24 ; 12)$.

I tempi di lettura dei preparati possono variare in relazione all'esperienza dell'operatore ed al tipo di campione.

$\underline{\text { Refertazione }}$

Il referto dell'esame coproparassitologico è NEGATIVO in caso di assenza di parassiti o POSITIVO in presenza di parassiti.

In caso di campione positivo, occorre riportare tutti i parassiti osservati; è necessario inserire un commento se uno o più dei parassiti identificati sono di norma considerati non patogeno $(9 ; 28$, pag. 18). Per ogni parassita identificato occorre riportare gli stadi vitali osservati (4, Appendice I, pag. 72; 36, pag. 170-171).

In ogni referto, il laboratorio deve esplicitare le ricerche effettuate sul campione in esame (4, Appendice I, pag. 72; 36, pag. 170-171)

In Allegato 2, che non è parte integrante di queste LGO, si riportano esempi di referti.

Il referto deve riportare: tutti i parassiti osservati; gli stadi vitali dei parassiti osservati; un commento se i parassiti osservati sono considerati di norma non patogeni; una esplicita indicazione delle ricerche effettuate sul campione (4, Appendice I, pag. 72; 9; 28, pag. 18; 35, pag. 170-171) $\rightarrow$ (A, VI).

\section{APPENDICE}

Livelli di prova, Forza delle raccomandazioni Livelli di prova

Prove di tipo I Prove ottenute da più studi clinici controllati randomizzati e/o da revisioni sistematiche di studi randomizzati.

Prove di tipo II Prove ottenute da un solo studio randomizzato di disegno adeguato.

Prove di tipo III Prove ottenute da studi di coorte non randomizzati con controlli concorrenti o storici o loro metanalisi.

Prove di tipo IV Prove ottenute da studi retrospettivi tipo caso-controllo o loro metanalisi.

Prove di tipo V Prove ottenute da studi di casistica ("serie di casi") senza gruppo di controllo.
Prove di tipo VI Prove basate sull'opinione di esperti autorevoli o di comitati di esperti come indicato in linee guida o consensus conferences o basata su opinioni dei membri del gruppo di lavoro responsabile di queste linee guida.

\begin{tabular}{ll}
\hline Forza delle raccomandazioni \\
\hline A & $\begin{array}{l}\text { Sta a indicare una forte raccomandazione a } \\
\text { favore dell'esecuzione di quella particola- } \\
\text { re procedura o test diagnostico. Indica una } \\
\text { particolare raccomandazione sostenuta da } \\
\text { prove scientifiche di buona qualità, anche } \\
\text { se non necessariamente di tipo I o II. }\end{array}$ \\
\hline B $\quad \begin{array}{l}\text { Quando si nutrono dei dubbi sul fatto che } \\
\text { quella particolare procedura o intervento } \\
\text { debba sempre essere raccomandata, ma si } \\
\text { ritiene che la sua esecuzione debba essere } \\
\text { attentamente considerata. }\end{array}$ \\
\hline C $\quad \begin{array}{l}\text { Quando esiste una sostanziale incertezza a } \\
\text { favore o contro la raccomandazione di ese- } \\
\text { guire la procedura o l'intervento. }\end{array}$ \\
\hline D o E Quando l'esecuzione della procedura non \\
è raccomandata.
\end{tabular}

\section{Metodologia}

Le Tabelle dei Livelli delle prove e della Forza delle raccomandazioni sono quelle elaborate dal Centro per la Valutazione dell'Efficacia dell'Assistenza Sanitaria (CeVEAS), sistema adottato dal Programma Nazionale Linee Guida (PNLG) coordinato dall'Istituto Superiore di Sanità e dall'Agenzia per i Servizi Sanitari Regionali (http://www.assr.it/plg/Manuale_PNLG.pdf).

Le Linee Guida sono state redatte utilizzando il seguente piano di lavoro:

1. Revisione della principale letteratura di settore.

2. Consultazione di Manuali ed Atlanti di autorevoli Parassitologi.

3. Consultazione dei principali "portali" di medicina on-line.

4. Studio di Linee Guida e Protocolli già esistenti in altri Paesi.

5. Studio di pubblicazioni con casistiche importanti, apparsi su riviste scientifiche riconosciute a livello nazionale e/o internazionale.

Alle prove derivate dai primi 4 punti (Manuali, Atlanti, Linee Guida e Protocolli) è stato assegnato il VI livello; alle prove derivanti da pubblicazioni con studi di casistiche è stato assegnato il V livello. In presenza di prove sia di $\mathrm{V}$ che di VI livello, il livello totale è stato assegnato in base alla mag- 
gioranza delle prove.

La Forza di tutte le raccomandazioni è sempre di tipo "A" data la generale concordanza di tutte le prove raccolte e l'elevato livello tecnico-scientifico dei pareri presi in esame.

La Bibliografia è numerata in ordine di citazione.

\section{Il Comitato di Studio per la Parassitologia ha deciso di revisionare queste Linee Guida entro 2 anni dalla pubblicazione.}

\section{BIBLIOGRAFIA}

1. De Carneri I. Parassitologia Generale e Umana; XI Ed., Casa Editrice Ambrosiana, Milano 1992.

2. Bernieri F, Crotti D, Galli D, Raglio A. Manuale illustrato di diagnostica parassitologica, Selecta Medica, Pavia 2001.

3. WHO. Bench Aids for the Diagnosis of Intestinal Parasites. WHO, Geneva 1994.

4. NCCLS. Procedures for the Recovery and Identification of Parasites from the Intestinal Tract; Approved Guideline. M 28-A. NCCLS, Waine PA USA 1997.

5. Petithory JC, Ardoin-Guidon F, Chaumeil C. Amibes et flagelles intestinaux. Amibes oculaires. Leur diagnostic microscopique. Cahier de Formation, Biologie medicale no. 11. Bioforma, Paris France1988.

6. WHO. Basic Laboratory Methods in Medical Parasitology. WHO, Geneva CH 1991.

7. Cheesbrough M. Medical Laboratory Manual for Tropical Countries; Vol I, Butterworth Ed 1987.

8. Rondanelli EG, Scaglia M. Atlante dei protozoi umani. Masson Ed., Milano 1993.

9. The Ontario Association of Medical Laboratories. Guidelines for the Collection and Testing for Ova and Parasites in Symptomatic Patients. CLP 018. OAML, North Yolk ON 1998. www.oaml.com/res pract.html

10. The Alberta Clinical Practice Guidelines Program. Laboratory Guideline for Ordering Stool Test for Investigation of Suspected Infectious Diarrhea. ACPGP, Edmonton AB 1997 (reviewed 2002).http://www.topalbertadoctors.org/guidelines/gu idelinespdf.aspx

11. Kargacin L. Laboratory Conference Highlights. Elaboration; Washington State Department of Health 2002; VII: issue 10, November/December.

12. College of Medical Laboratory Technologists of Ontario. Workload Guidelines for Parasitology Technologists. CMLTO, Toronto ON 1999. http://www.cmlto.com/quality assurance/mlt prac$\underline{\text { tice guidelines/default.asp }}$

13. British Columbia Medical Association. Guidelines \& Protocols Advisory Committee. Investigation of Suspected Infectious Diarrhea G\&P 2003-062. BCMA, Victoria BC 2000 (reviewed 2003). www.hlth.gov.bc.ca/msp/protoguides/gps/diarrhea.pdf

14. Hanson KL, Cartwright CP. Use of an Enzyme Immunoassay Does Not Eliminate the Need To Analyze Multiple Stool Specimens for Sensitive Detection of Giardia lamblia. J Clin Microbiol 2001; 39: 474-7.

15. Marti H, Koella JC. Multiple Stool Examinations for Ova and Parasites and Rate of False-Negative Results. J Clin Microbiol 1993; 31: 3044-5.

16. Parija SC, Srinivasa H. Viewpoint: The neglect of stool microscopy for intestinal parasites and possible solutions. Trop Med Public Health1999; 4: 522-4.

17. Clavel A, Arnal AC, Sanchez EC, Varea M, Castillo FJ, Ramirez de Ocariz I, Quilez J, Cuesta J. Evaluation of the optimal number of faecal specimens in the diagnosis of cryptosporidiosis in AIDS and immunocompetent patients. Eur J Clin Microbiol Infect Dis 1995; 14: 46-9.

18. Garcia LS Practical Guide to Diagnostic Parasitology. American Society for Parasitology, Washington DC 1999.

19. Garcia LS Diagnostic Medical Parasitology, IV Ed. American Society for Parasitology, Washington DC 2001.

20. Estevez EG, Levine JA. Examination of preserved stool specimens for parasites: lack of value of the direct wet mount. J Clin Microbiol 1985; 22: 666-7.

21. Neimeister R, Logan AL, Egleton JH, Kleger B. Evaluation of direct wet mount parasitological examination of preserved fecal specimens. J Clin Microbiol 1990; 28: 1082-4.

22. Bernieri F, Galli D, Viti F, “et al.”. Parassitosi enteriche autoctone. Microbiologia Medica 1994; 9: 415-8.

23. Bernieri F, Galli D, Giordano S, "et al.”. Indagine nazionale AMCLI-CoSP sulla diffusione delle parassitosi intestinali. Microbiologia Medica 1996; 11: 463-72.

24. Garcia LS. Diagnostic Medical Parasitology: An Update. Clin Microb Newsletter 1994; 16: 105-10.

25. Kellogg JA, Elder CJ. Justification for Use of a Single Trichrome Stain as the Sole Means for Routine Detection of Intestinal Parasites in Concentrated Stool Specimens. J Clin Microbiol 1999; 37: 835-7.

26. Garcia LS, Shimizu RY. Medical Parasitology: Update on Diagnostic Techniques and Laboratory Safety. Lab Med, 1993; 24: 81-8.

27. Lainson R, da Silva BAM.. Intestinal Parasites of Some Diarrhoeic HIV-Seropositive Individuals in North Brazil, with Particular Reference to Isospora belli Wenyon, 1923 and Dientamoeba fragilis Jepp \& Dobell, 1918. Mem Ist Oswaldo Cruz 1999; 94: 611-3.

28. Health Protection Agency. Standard Operating procedure. Investigation of Specimen Other Than Blood for Parasites. BSOP 31i.1. Health Protection Agency, UK 2003.

29. Arakaki T, Iwanaga M, Kinjo F, “et al.”. Efficacy of Agar Plate Culture in Detection of Strongyloides stercoralis Infection. J Parasitol 1990; 76: 425-8.

30. Koga K, Kasuya S, Khamboonruang C, "et al.”. A Modified Agar Plate Method for Detection of Strongyloides stercoralis. Am J Trop Med Hyg 1991; 45: 518-21.

31. De Kaminsky RG. Evaluation of Three Methods for Laboratory Diagnosis of Strongyloides stercoralis Infection. J Parasitol 1993; 79: 277-80.

32. Assefa T, Woldemichael T, Seyoum T. Evaluation of the modified Baermann's method in the laboratory diagnosis of Strongyloides stercoralis. Ethiop Med J 1991; 29:193-8.

33. Sato Y, Kobayashi J, Toma H, Shiroma Y. Efficacy of stool examination for detection of Strongyloides infection. Am J Trop Med Hyg 1995; 53: 248-50.

34. Jongwutiwes S, Charoenkorn M, Sitthichareonchai P, Akaraborvorn P, Putaporntip C. Increased sensitivity of routine laboratory detection of Strongyloides stercoralis and hookworm by agar-plate culture. Trans R Soc Trop Med Hyg 1999;93: 398-400.

35. MacPherson D.W., McQueen R. Cryptosporidiosis: Multiattribute Evaluation of Six Diagnostic Methods. J Clin Microbiol 1993; 31: 198-202.

36. W.H.O. Manual of Basic Techniques for a Health Laboratory. W.H.O, Geneve CH 1980. 


\section{ALLEGATI}

I seguenti allegati non sono parte integrante delle Linee Guida ma vengono riportati come sussidio all'applicazione delle stesse.

\section{Allegato 1}

\section{ESEMPI DI ISTRUZIONI PER I PAZIENTI}

Tabella 1. Istruzioni per la raccolta delle feci

ESAME COPROPARASSITOLOGICO STANDARD

N.B.: si raccomanda di raccogliere 3 campioni di feci emesse a gionni alterni

1) Le feci vanno raccolte su una superficie asciutta e pulita, tipo una padella da letto, oppure un foglio di cartone o giornale ripiegato, o un sacchetto di plastica, posti sotto il copri water.

N.B.: le feci non vanno contaminate né con le urine né con l'acqua del water.

2) Le feci vanno prelevate in punti diversi dell'intera evacuazione. Trasferire in un adeguato contenitore (di plastica, fornito dal laboratorio o acquistato in farmacia), utilizzando una spatola o una bacchetta di legno o una posata di plastica, una quantità di feci pari almeno al volume di una grossa noce.

N.B.: se le feci sono non formate/diarroiche raccogliere almeno 5-10 $\mathrm{ml}$ di materiale fecale.

3) Chiudere molto bene il contenitore ed etichettarlo con cognome e nome, data di nascita, data e ora dell'emissione delle feci.

4) Consegnare il campione al laboratorio entro 2-4 ore dalla raccolta oppure conservarlo in frigorifero per non più di 24 ore.

ATTENZIONE: se le feci sono liquide il campione deve pervenire al laboratorio entro 30-60 minuti dalla loro emissione!

\section{PRECAUZIONI IMPORTANTI}

a) Alcuni giorni prima e durante il periodo della raccolta delle feci non fare uso di lassativi, antidiarroici, antimicrobici, o di altre sostanze interferenti come bario, bismuto, oli minerali.

b) Alcuni giorni prima e durante il periodo della raccolta delle feci si consiglia di seguire un regime dietetico che prevede di evitare: legumi e frutta secca, frutti e verdure a cuticola resistente (pesche, albicocche, pomodori, pere, fragole, fichi), carote e banane.

Tabella 2. Istruzioni per l'esecuzione dello Scotch Test

ESECUZIONE DELLO SCOTCH TEST PER LA RICERCA SPECIFICA DI

ENTEROBIUS VERMICULARIS (OSSIURI)

\section{N.B.: si raccomanda di eseguire 3 scotch test preferibilmente a giorni alterni.}

\section{ATTENZIONE:}

- la raccolta deve essere eseguita al momento del risveglio mattutino, prima che il soggetto defechi e si lavi

- utilizzare nastro adesivo (scotch) trasparente

- ritirare il materiale necessario (vetrini e abbassalingua) in laboratorio

1) Tagliare con le forbici un pezzo di nastro adesivo $(5-6 \mathrm{~cm})$ un poco più corto del vetrino fornito dal laboratorio.

2) Con l'aiuto di un abbassalingua (o del manico di un cucchiaio) appoggiare il nastro adesivo sull'orifizio anale comprimendo bene sulle pliche perianali per circa 15-20 secondi.

3) Staccare il nastro adesivo dall'orifizio anale ed applicarlo ben steso sul vetrino.

4) Identificare il vetrino con cognome e nome e data del prelievo.

5) Consegnare il campione al laboratorio entro 2-4 ore dal prelievo oppure conservarlo in frigorifero per non più di $24-48$ ore. 
Tabella 3. Istruzioni per la raccolta delle feci

RICERCA SPECIFICA DI STRONGYLOIDES STERCORALIS

\section{N.B.: si raccomanda di raccogliere 3 campioni di feci emesse a giorni alterni}

1) Le feci vanno raccolte su una superficie asciutta e pulita, tipo una padella da letto, oppure un foglio di cartone o giornale ripiegato, o un sacchetto di plastica, posti sotto il copri water.

N.B.: le feci non vanno contaminate né con le urine né con l'acqua del water.

2) Le feci vanno prelevate in punti diversi dell'intera evacuazione. Trasferire in un adeguato contenitore (di plastica, fornito dal laboratorio o acquistato in farmacia), utilizzando una spatola o una bacchetta di legno o una posata di plastica, una quantità abbondante di materiale fecale, corrispondente al peso di almeno 30-40 grammi. Ciò equivale a una quantità di feci pari al volume di un mandarino.

3) Chiudere molto bene il contenitore ed etichettarlo con cognome e nome, data di nascita, data e ora dell'emissione delle feci.

4) Consegnare il campione al laboratorio entro 6-12 ore dalla raccolta.

\section{PRECAUZIONI IMPORTANTI}

Alcuni giorni prima e durante il periodo della raccolta delle feci non fare uso di lassativi, antidiarroici, antimicrobici, o di altre sostanze interferenti come bario, bismuto, oli minerali.

Alcuni giorni prima e durante il periodo della raccolta delle feci si raccomanda di seguire un regime dietetico che prevede di evitare: legumi e frutta secca, frutti e verdure a cuticola resistente (pesche, albicocche, pomodori, pere, fragole, fichi), carote e banane.

Allegato 2

ESEMPI DI REFERTI

\section{Referto 1}

Cognome e Nome: D. G.

Materiale: Feci formate

Esame richiesto: $\quad$ Esame coproparassitologico

Esito: Negativo

Esami eseguiti: $\quad$ Esame Microscopico Diretto; Esame Microscopico dopo

Concentrazione (FEA); Colorazione Tricromica.

\section{Referto 2}

Cognome e Nome: A. R.

Materiale: $\quad$ Feci diarroiche

Esame richiesto: $\quad$ Esame coproparassitologico

Esito: $\quad$ Positivo

Identificazione: $\quad$ Trofozoiti e Cisti di Giardia intestinalis

Esami eseguiti: $\quad$ Esame Microscopico Diretto; Esame Microscopico dopo

Concentrazione (FEA); Colorazione di Giemsa.

\section{Referto 3}

\begin{tabular}{ll}
\hline Cognome e Nome: & D. C. \\
\hline Materiale: & Feci non formate \\
\hline Esame richiesto: & Esame coproparassitologico per ricerca di Strongyloides stercoralis \\
\hline Esito: & Positivo \\
\hline Identificazione: & Larve rabditoidi di Strongyloides stercoralis \\
\hline Esami eseguiti: & Esame Microscopico Diretto; Metodo di Baermann. \\
\hline
\end{tabular}


Referto 4

Cognome e Nome: $\quad$ F. B.

Materiale: Feci formate

Esame richiesto: $\quad$ Esame coproparassitologico

Esito:

Positivo

Identificazione:

1) Uova di Taenia spp

2)Cisti di Entamoeba coli

Esami eseguiti: $\quad$ Esame Microscopico Diretto; Esame Microscopico dopo

Concentrazione (FEA); Colorazione Tricromica.

Nota:

Entamoeba coli è di norma considerata NON patogena

\section{Referto 5}

Cognome e Nome: P. C.

Materiale:

Feci diarroiche

Esame richiesto:

Esame coproparassitologico

Esito:

Positivo

Identificazione:

Esami eseguiti:

Trofozoiti di Dientamoeba fragilis

Esame Microscopico Diretto; Esame Microscopico dopo

Concentrazione (FEA); Colorazione di Giemsa.

\section{Referto 6}

Cognome e Nome:

Materiale:

L. D.

Esame richiesto:

Esito:

Feci diarroiche

Identificazione:

Esame coproparassitologico

Esami eseguiti:

Positivo

Trofozoiti di Entamoeba histolytica/dispar

\begin{tabular}{ll}
\hline Nota: & $\begin{array}{l}\text { Entamoeba histolytica (patogena) e Entamoeba dispa } \\
\text { sono tra di loro morfologicamente indistinguibili. }\end{array}$ \\
\hline
\end{tabular}

Esame Microscopico Diretto; Esame Microscopico dopo

Concentrazione (FEA); Colorazione Tricromica.

\section{Referto 7}

\begin{tabular}{ll}
\hline Cognome e Nome: & V. C. \\
\hline Materiale: & Scotch Test \\
\hline Esame richiesto: & Ricerca Ossiuri \\
\hline Esito: & Positivo \\
\hline Identificazione: & Uova di Enterobius vermicularis \\
\hline
\end{tabular}

\section{Francesco Bernieri}

Direzione Generale Sanità / U.O. Qualità e

Appropriatezza dei Serv. Sanitari

Via Pola 11, Milano.

Tel 0267653134 ,

E-mail: fbernieri@dgsan.lombardia.it francesco.bernieri@aliceposta.it 